\title{
Colonel Blotto Games with Favoritism: Competitions with Pre-allocations and Asymmetric Effectiveness
}

\author{
DONG QUAN VU, Univ. Grenoble Alpes, CNRS, Inria, Grenoble INP, LIG, France \\ PATRICK LOISEAU, Univ. Grenoble Alpes, Inria, CNRS, Grenoble INP, LIG, France
}

CCS Concepts: • Theory of computation $\rightarrow$ Exact and approximate computation of equilibria.

Additional Key Words and Phrases: Blotto games; all-pay auctions

\section{ACM Reference Format:}

Dong Quan Vu and Patrick Loiseau. 2021. Colonel Blotto Games with Favoritism: Competitions with Preallocations and Asymmetric Effectiveness. In Proceedings of the 22nd ACM Conference on Economics and Computation (EC '21), July 18-23, 2021, Budapest, Hungary. ACM, New York, NY, USA, 2 pages. https://doi.org/ $10.1145 / 3465456.3467527$

\section{EXTENDED ABSTRACT}

The Colonel Blotto game, first introduced by [1], is a famous resource allocation game: two players, $\mathrm{A}$ and $\mathrm{B}$, compete over $n$ battlefields by simultaneously distributing their limited budget of resources to maximize the aggregate values from the battlefields that they win. It has attracted great attention in the literature and has been successfully applied to a large range of practical situations (e.g., [6, 8]).

In many of these applications, however, the classical winner-determination rule of the Colonel Blotto game (i.e., giving the whole battlefield's value to the player who has the higher allocation and zero to the other) is too restrictive because a player might have an advantage over some battlefields. We refer to this as favoritism. There can be two basic types of favoritism: first, players may have resources committed to battlefields before the game begins-called the pre-allocations-, which then add up to the allocations to determine the winner in each battlefield; second, the resources effectiveness may not be the same for the two players, and may vary across battlefields.

To account for this, we propose and analyze an extension of the Colonel Blotto game with a new winner-determination rule: in battlefield $i \in\{1, \cdots, n\}$, if the allocations of Players A and B are $x^{A}$ and $x^{B}$ respectively, Player A wins if $x^{A}>q_{i} \cdot x^{B}-p_{i}$ and Player B wins otherwise. Here, $p_{i} \in \mathbb{R}$ represents the difference between the pre-allocations that players have at battlefield $i$; thus, if $p_{i}>0$, Player A has a larger pre-allocation and otherwise, Player B has this advantage. On the other hand, $q_{i}$ represents the asymmetry in the effectiveness of players' resources: each unit of Player B's resource is worth $q_{i}$ units of Player A's resource; if $0<q_{i}<1$, Player A's resource is more effective than that of Player B and reversely if $q_{i}>1$. We term this extended game the Colonel Blotto game with favoritism (F-CB).

In this work, we focus on characterizing and computing Nash equilibria of the F-CB game. Characterizing equilibria is also the main focus of the Blotto game literature [2-5, 7]. This line of research follows a common approach which starts by looking for a set of optimal univariate distributions of the game (often based on the equilibria of all-pay auctions), and then constructing

Permission to make digital or hard copies of part or all of this work for personal or classroom use is granted without fee provided that copies are not made or distributed for profit or commercial advantage and that copies bear this notice and the full citation on the first page. Copyrights for third-party components of this work must be honored. For all other uses, contact the owner/author(s).

EC '21, fuly 18-23, 2021, Budapest, Hungary

(c) 2021 Copyright held by the owner/author(s).

ACM ISBN 978-1-4503-8554-1/21/07.

https://doi.org/10.1145/3465456.3467527 
$n$-variate joint distributions whose realizations satisfy the budget constraints. Constructing such joint distributions, however, is notoriously challenging and equilibria are only successfully characterized in several restricted instances. We analyze the F-CB game by following a similar pattern and make three main contributions:

(1) First, we consider the closely related model of all-pay auctions with favoritism and completely characterize its equilibrium. While this game was studied in prior works, our result fills a gap in the literature.

(2) We prove the existence of a set of optimal univariate distributions of the F-CB game and give a construction thereof. The main challenge is that it is equivalent to finding a fixed point, but for a complex two-dimensional function for which standard existence results fail to apply. We overcome this obstacle by drawing tools from topology and carefully tailoring them to our particular problem. We deduce from this core result the equilibrium of the F-CB game in several special cases. For other cases, we show that, by applying the rescaling technique of [9], we can obtain an approximate equilibrium of the F-CB game with negligible approximation error when the number of the battlefields is large. Finally, for any parameter configuration, we also immediately obtain the exact equilibrium of an important relaxed variant of F-CB where budget constraints are only required to hold in expectation (called the General Lotto game with favoritism).

(3) We propose an algorithm that efficiently finds an approximation of the proposed optimal univariate distributions with an arbitrarily small error. This improves the scalability of our results upon the naive solution for exact computation (which is exponential in the number of battlefields). Our algorithm is based on approximately solving the two-dimensional fixedpoint problem by a dichotomy procedure using a generalization of the intermediate value theorem with the notion of winding number of parametric curves.

Finally, we conduct several experiments illustrating the effect of favoritism in the F-CB game. A full version of our paper is available at https://arxiv.org/abs/2106.00617.

\section{ACKNOWLEDGMENTS}

This work has been partially supported by MIAI @ Grenoble Alpes (ANR-19-P3IA-0003) and by the French National Research Agency through grant ANR-19-CE48-0018.

\section{REFERENCES}

[1] E Borel. 1921. La théorie du jeu et les équations intégrales à noyau symétrique. Comptes rendus de l'Académie des Sciences 173, 1304-1308 (1921), 58.

[2] E Borel and J Ville. 1938. Application de la théorie des probabilités aux jeux de hasard. Gauthier-Villars. Original edition by Gauthier-Villars, Paris, 1938; reprinted at the end of Théorie mathématique du bridge à la portée de tous, by E. Borel \& A. Chéron, Editions Jacques Gabay, Paris.

[3] Oliver Gross. 1950. The symmetric Blotto game. Technical Report. US Air Force Project RAND Research Memorandum.

[4] Oliver Gross and Robert Wagner. 1950. A continuous Colonel Blotto game. Technical Report. RAND project air force Santa Monica CA.

[5] Dan Kovenock and Brian Roberson. 2020. Generalizations of the General Lotto and Colonel Blotto games. Economic Theory (2020), 1-36.

[6] Roger B Myerson. 1993. Incentives to cultivate favored minorities under alternative electoral systems. American Political Science Review 87, 4 (1993), 856-869.

[7] Brian Roberson. 2006. The Colonel Blotto Game. Economic Theory 29, 1 (2006), 1-24.

[8] Galina Schwartz, Patrick Loiseau, and Shankar S Sastry. 2014. The heterogeneous Colonel Blotto game. In Proceedings of the 7th International Conference on Network Games, Control and Optimization (NetGCoop). 232-238.

[9] Dong Quan Vu, Patrick Loiseau, and Alonso Silva. 2020. Approximate Equilibria in Generalized Colonel Blotto and Generalized Lottery Blotto Games. arXiv:1910.06559v2. 